\title{
Localization for Emergency Sensor Networks
}

\author{
Horacio Sanson \\ Global Information and Telecommunication Studies \\ Waseda University \\ Tokyo, Japan \\ Email: hsanson@moegi.waseda.jp
}

\author{
Matsumoto Mitsuji \\ Global Information and Telecommunication Institute \\ Waseda University \\ Tokyo, Japan \\ Email: mmatsumoto@waseda.jp
}

\begin{abstract}
Communication systems are essential in the coordination and planning of emergency and disaster relief operations (fire, earthquake, terrorism). Unfortunately, existing systems often provide minimal communication infrastructure for suplying information about the nature or the extend of an emergency or disaster area. Wireless Sensor Networks are a promising method for providing real-time feedback from disaster sites to rescue personnel. In this paper we evaluate current localization methods for WSN's in scenarios with characteristics similar to those common in a disaster area. We find that common assumptions in current location schemes don't apply in a disaster scenario and the expected behavior of the localization method changes. Even thought the expected behavior changes we show that current proposed localization methods maybe fit for rescue and disaster relief applications. Finally we mention deficiencies and improvements for this localization methods.
\end{abstract}

\section{INTRODUCTION}

The first widely known example of the use of wireless communication technologies to find victims in a disaster area was the Wireless Response Team [12]. They tried to use cellular phone and pager signals to detect the location of victims trapped inside the rubble after the September 11 World Trade Center attack. The report presented by WERT had several recommendations and key learnings for improving localization techniques in rescue operations. Based on the characteristics of the disaster area described in the WERT report is clear that current localization methods GPS (Global Positioning System) and Cellular based (E911) where useless in the aid to detect victims inside the rubble due to several factors:

1) The area was large ( 14 acres, 7 stories tall and 7 stories down) and the number of expected victims was big (Thousands).

2) Occlusion, reflections and multipath effects limit the usefulness of GPS in indoor or dense environments, situation that was common in the disaster area of the WTC.

3) The cellular network was severely affected by the disaster ( 3 cells were destroyed and 173 were affected by network or power connectivity). This limited the use of cellular technology to detect signals from cell phones and pagers.

4) The use of portable equipment (Spectral analyzers, Cells on Wheels, etc..) to detect RF signals from cell phones and pagers was also limited by the following factors: a) Hostile RF environment due to dense concrete and metal. RF signals were scattered limiting the value of directional antennas.

b) Bad weather and heavy rain that increased the capacity of concrete to absorb RF signals.

c) There was interference between the equipment used by WERT and that used by the rescue teams. Was difficult to determine if the RF signals detected were from victims in the ruble or rescue team members.

5) Limited access and mobility due to hazardous environment, most access was restricted to the perimeter of the disaster area. The location of COWs (Cells on Wheels) and paging devices were far away from the disaster area than ideal since the area was filled with heavy equipment from rescue teams and low availability of power supply.

Wireless Sensor Networks (WSN) [16], networks of small sensor devices interconnected via wireless links, promise to help solve this and future problems. The idea of small sensors connected via wireless links that can provide information about the environment in real time assures that rescue team members can make decisions based on more complete information. The Wireless Sensor Networks are widely recognized as a privileged way to provide information in situations where less robust and less flexible fixed infrastructures are temporarily inoperable [17]. In this work we study WSN's as means to aid in relief and rescue operations duritig a major disaster events. We focus our studies in localization protocols for WSN's because location information is crucial in this kind of situations, for example tracking the movement of a forestal] fire or locating trapped survivors in a disaster area.

First we must set some requiremets to considered before using a localization method for wireless sensor network to detect victims or hazardous substances inside a disaster area. Based on the characteristics presented by WERT we see that the dimensions of the disaster area is large and hazardous. This requires a very large scale sensor network so the localization methods must be scalable and robust in case of node failures. The localization method must be robust to ranging errors to compensate multipath and fading effects that are amplified by several factors in a disaster area. Finally because of the hazardous nature of the disaster area and limited access and mobility, during the rescue operations of september 11 the 
WERT team members could only operate on the perimeter of the disaster area which indicates that anchor nodes may be only available at the edges of the sensor network and in low quantities.

\section{LOCALIZATION METHODS FOR WSN'S}

There is a lot of research in localization methods for wireless sensor networks as seen in Table I. The Centroid method [3] is the most basic method. In the Centroid method anchor nodes with known positions broadcast a beacon message with their position to the network. Each sensor node hears all beacons from nearby anchors and when it has more than three or more, it estimates it's own location as the centroid of all beacons with the formula:

$$
\left(X_{e s t}, Y_{e s t}\right)=\left(\frac{X_{1}+\ldots+X_{k}}{k}, \frac{Y_{1}+\ldots+Y_{k}}{k}\right)
$$

Where $X_{\text {est }}$ and $Y_{\text {est }}$ are the estimated coordinates of the sensor node and $X_{1: k}$ and $Y_{1: k}$ are the coordinates of all $k$ anchor nodes heard by the sensor node.

The APIT method [13] improves over the centroid estimation by narrowing down the possible area in which a node may reside by using Point-In-Triangle tests. Each node in the APIT method repeats this PIT test for all three audible anchor combinations or until a threshold is achieved. At this point APIT calculates the estimate position as the center of gravity of the intersecting area of all triangles the node resides in using an aggregation algorithm. This two methods, Centroid and APIT, require a dense population of anchor nodes distributed over the whole network because direct communication between sensor nodes and anchors is required.

DV-Hop, DV-Distance and Euclidean form the family of methods proposed in [2]. This methods differ in the way distances from anchors to sensor nodes are estirnated. All three use multi-hop communication to propagate distance information with beacon messages from hop to hop to all the network. In DV-Hop the beacon propagates hop count information from anchors to sensor nodes. This hop count value is stored in each sensor with the position and id of the originating anchor. During this propagation the beacon may reach other anchors in the network, at this point anchors calculate an average hop count $h_{s i z e_{i}}$ with information from other anchors as:

$$
h_{\text {size }}=\frac{\sum \sqrt{\left(X_{i}-X_{j}\right)^{2}+\left(Y_{i}-Y_{j}\right)^{2}}}{\sum h_{i}}, i \neq j
$$

This $h_{\text {size }}$ is then broadcasted to the whole network and each sensor node that receives it converts it's hop count value to distances as $h_{\text {size }_{i}}$ *hopCount $i$ for all anchors $i$ it heard. DV-Distance is similar to DV-Hop but instead of hop counts the accumulated distance estimated from hop to hop is propagated instead of hop counts. Euclidean is more complex and propagates the real euclidean distance between sensors and anchors. With this estimated distances and the location of the anchor nodes, each sensor node can estimate it's own location via lateration (intersection of circles) or min-max (intersection of squares).
TABLE ]

LIST OF LOCALIZATION METHODS FOR WIRELESS SENSOR NETWORKS.

\begin{tabular}{|l|c|c|c|}
\hline Name & Distributed & Range-Free & Mulit-Hop \\
\hline Convex [1] & no & yes & no \\
\hline MDS-MAP [10] & no & yes & no \\
\hline Centroid [3] & yes & yes & no \\
\hline APIT [13] & yes & yes & no \\
\hline Arnorphous [8] & yes & yes & yes \\
\hline DV-Hop [2] & yes & yes & yes \\
\hline DV-Distance [7] & yes & no & yes \\
\hline DV-Euclidean [2] & yes & no & yes \\
\hline Hop-Terrain [4] & yes & yes & yes \\
\hline AHLoS [5] & yes & no & no \\
\hline n-Hop Lateration [7] & yes & no & no \\
\hline MCL [9] & yes & yes & no \\
\hline
\end{tabular}

The Amorphous method is similar to DV-Hop, the only difference is the way the average hop size is calculated. In the Amorphous approach instead of anchors calculating an average and broadcasting a second wave the hop size is calculated with the Kleinrock formula [14] that says that the hop size depends only on the local neighborhood density nlocal, not the total number of sensors.

$$
h_{\text {size }}=r\left(1+e^{-n_{\text {local }}}-\int_{-1}^{1} e^{-\frac{n_{\text {icenal }}\left(\arccos t-t \sqrt{1-t^{2}}\right.}{\pi}} d t\right)
$$

Finally Hop-Terrain is identical to DV-Hop but adds a refinement phase after the initial location estimation takes place to improve the estimated distances of nodes.

The AHLoS method [5] defines an iterative multilateration method. Once the network is deployed, anchor nodes broadcast. beacons with their positions to their neighbors. Neighboring sensor nodes with unknown location measure their separation from their anchor nodes and use the beacons position information to infer it's own position. When this unknown sensor nodes estimate it's position, it becomes an anchor and broadcasts it's estimated position to nearby unknown nodes. This process continues until all sensor nodes get an estimate of their position.

In some situations a sensor node may not have enough neighbors with known position to estimate it's own. In this case the author defines collaborative lateration where two hop neighbor information may be used to infer location of sensor nodes. Also without relative large beacon densities, the iterative process may not converge in sparse regions of the network.

In [7] the same author improves on AHLOS and generalizes the collaborative lateration to n-Hop lateration. In this improved method nodes are grouped in subtrees and enforced within the subtree to estimate their positions in turn in a fixed sequence to enhance convergence.

\section{SIMULATION ENVIRONMENT}

During the WERT operations the area of disaster was of 14 acres $\left(64749.70 \mathrm{~m}^{2}\right)$ with thousands of victims $(40,000)$ so a large scale network composed of thousands of sensor nodes to cover the whole area is needed. This requirement makes centralized approaches like Convex and MDS inappropriate since 


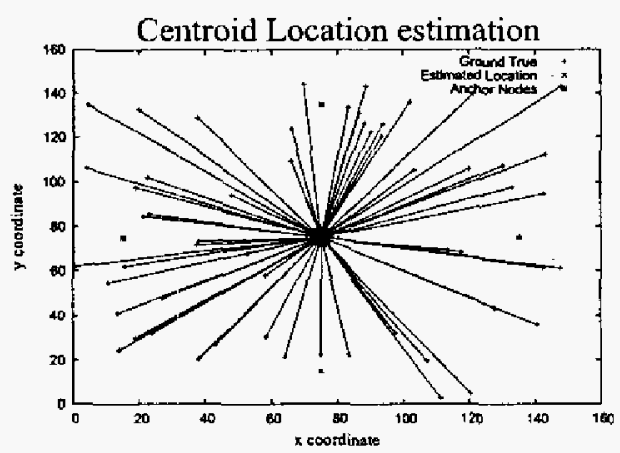

Fig. 1. Centroid Estimation Method: All estimated locations are pushed to the center of the simulation area. Four anchor nodes at the perimeter. The lines represent the error in the location estimate

centralized approaches can't deal with topology changes due to mobility or node failures because of the high communication costs and inherent delay and are not scalable in processing as node density increases.

As we mention WERT members were restricted to the perimeter of the area so in our simulations we assume all anchor nodes are at the perimeter of the simulation area. This assumption renders the centroid and APIT localization methods unusable. Even if the anchor nodes have large transmission capability that covers the whole area, the location estimates based on the centroid equation 1 would push all estimated locations to the center of the simulation area generating large errors in the estimates, see figure 1

The distributed approaches by Niculescu and Savvides seem to be more appropriate for localization in wireless sensor networks [15] but still we need to study their performance under the constraints imposed if deployed in a disaster area. We chose to evaluate all three methods proposed by Niculescu: DVHop, DIDistance and Euclidean and from Savvides we only evaluate the $n$-Hop Mutilateration method since it is an improvement and generalization of the $A H L O S$ method.

For evaluation purposes we use the OMNet + discrete event simulator [11] and it's Mobility Framework extension. All simulations are performed in a $350 \times 350$ square area with anchor nodes in the perimeter and nodes randomly distributed in the square area. The physical layer is free of collisions so all messages arrive to their destinations and the radio propagation is simulated with different ranging errors. For this we estimate ranging distances tretween nodes and add noise to the distance taking a random value from a normal distribution with the real distance as mean and standard deviation in the range of $0 \%-50 \%$ of the mean. All simulations are run five times with different random generator seeds and the resulting statistics are averaged over the five runs to account for peculiarities in the network topology.

\section{SIMULATION RESULTS}

In Jocation estimation once the distance to known reference is known we can estimate the location using several methods

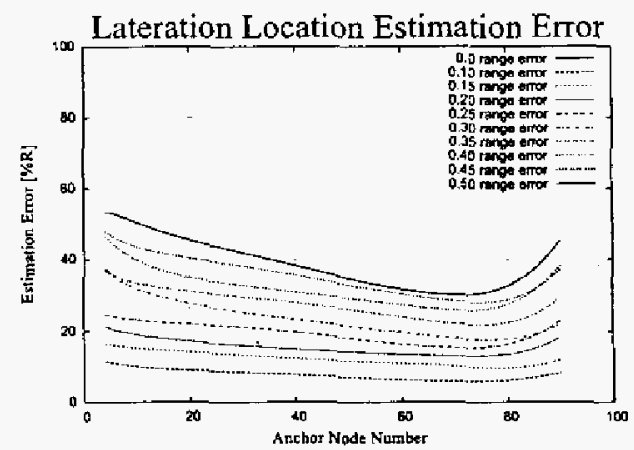

Fig. 2. Lateration Estimation Firnor

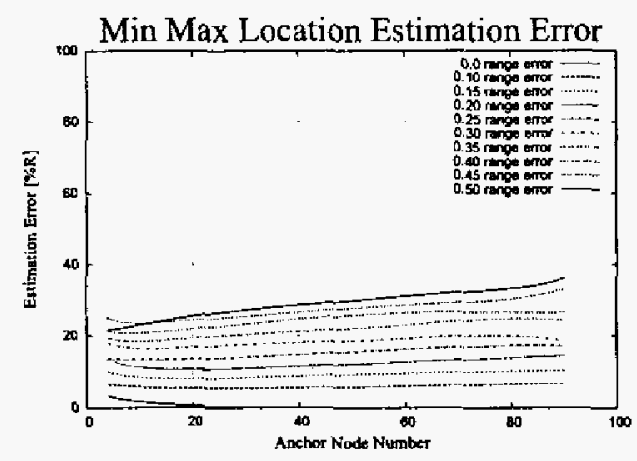

Fig. 3. Min Max Estimation Error

like trilateration (intersection of circles) or min-max (intersection of squares). Our simulations show that trilateration, figure 2 , is very accurate in the location estimates for $0 \%$ ranging errors but as ranging errors increase the location estimate acurracy is greatly affected. On the other hand minmax, figure 3, is less accurate; it presents errors even with $0 \%$ ranging errors but it is more robust as ranging errors increase. Trilateration presents a little improvement as anchor node density increases but min max presents an slight increase in estimation error. Some previous work show that min max fails for nodes outside the convex hull formed by the anchor nodes, this is not the case in our simulations since all anchor nodes are at the perimeter of the area.

From the previous discussion we chose to use min max for location estimation in all our simulations. The accuracy deficiency of min max is not too large compared to trilateration and min max presents more benefits like easy implementation and lower complexity. Also being robust to ranging errors is a quality we desire in a disaster area where occlusion, fading and multipath effects to RF signals are amplified by the environment as stated before.

We developed simulations in OMNet++ for DVHop, DVDistance, Euclidean and n-Hop Multilateration localization methods following as much as possible the description of the original papers. We found that sone of the sequirements imposed by a disaster relief situation change the expected 


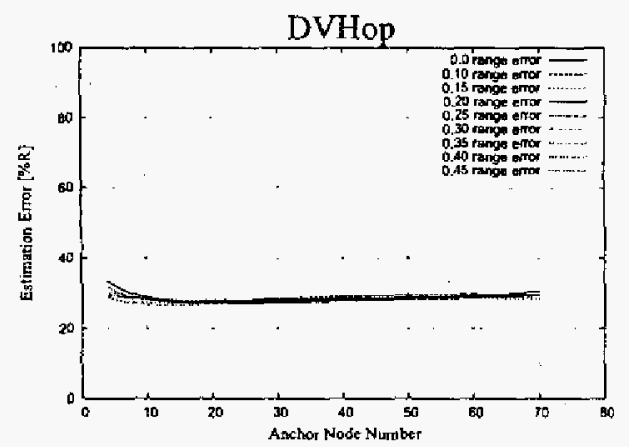

Fig. 4. DVHop Location Estimation Error

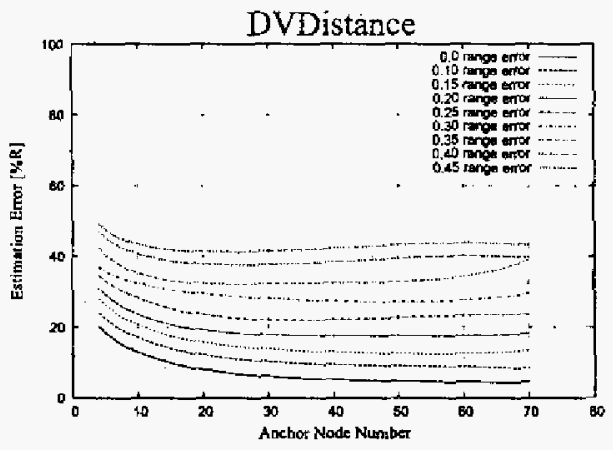

Fig. 5. DVDistance Lacation Estimation Error

behavior observed by the authors and in some cases simplified the implementation of the methods.

For example in the Euclidean method the solution of the diagonal in the quadrilateral gives two possible distance estimates. Niculescu offer several methods to chose one of the two solutions based on more neighbors information or simply guess. We found that simply choosing the larger of both solutions gives better overall location estimates. This is due to the fact that anchor nodes are at the perimeter and the beacons propagation method ensures that most sensor nodes with unknowns will be farther from anchors.

In the $\mathrm{n}$-Hop multilateration approach messages between nodes are exchanged any time a sensor obtains a new location estimate, this process repeats until the change in the location estimate is small. Unfortunately if this process is not controlled the nodes where the iterative process initiates would nun several iterations before the first estimated locations propagate through the whole network. To avoid this the author forces all nodes to estinate their locations in a sequential manner setting timers to the sensor nodes, only when the timer ends the node estimates it's location an propagates the estimated location. The timer is set to a value long enough to allow the propagation gradient to cover the whole network.

To abtain the value of this timer, Savvides uses a Distributed Depth First Search (DDFS) algorithrn over the network. In our case we simply send a single message and estimate the time

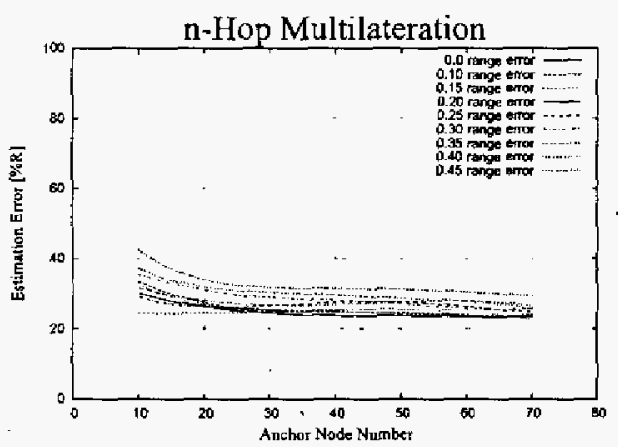

Fig. 6. n-Hop Multilateration Location Estimation Error

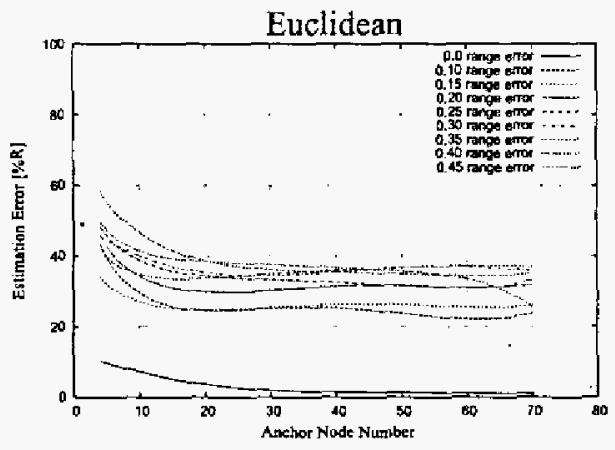

Fig. 7. Euclidean Location Estimation Error

it takes to reach from one side to the other side and use it as the timer value.

Another advantage of having anchor nodes, at the edges is that this anchor nodes may be mobile. If anchor nodes are mobile different sensors in the network may detect the anchor at different locations; each time the same anchor can be taken as two different anchors so low density of anchor nodes can be compensated by anchor nodes mobility.

Figures 4 to 7 show the simulation results. Each point is an average of five runs, the vertical axis is the location estimation error as a fraction of the radio range and the horizontal axis is the anchor node density varied from 4 nodes to 70 nodes. All graphs present several curves, each one corresponding to a different ranging error in the distance estimates between neighbors with values from $0.0 \%$ to $45 \%$ the real distance.

The first thing to note in all graphs is that different from other studies the anchor node density does not affect the location estimation error. Other studies mention that anchor node density is an important factor affecting accuracy of the localization method. Since our anchor nodes are all at the perimeter of the area, increasing the anchor node desity has little effect on the location estimate. One exception is the n-Hop multilateration method that has no location estimates for densities below 10 anchor nodes. This is due to the fact that at such low densities the conditions for atomic lateration that initiates the iterative process are not met and as such the 


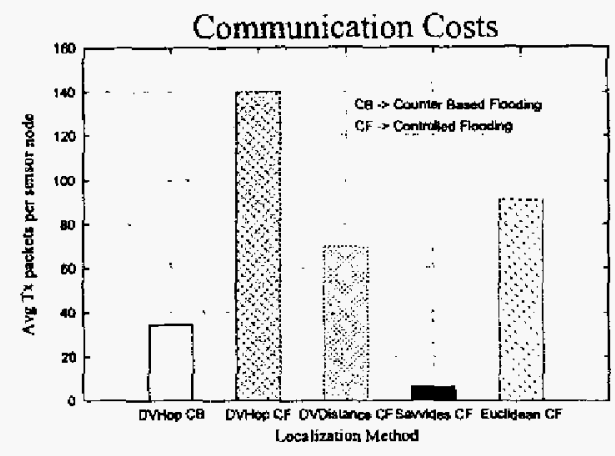

Fig. 8. Communication costs in packets transmitted

process doesn't even start and all sensor nodes are unable to get location estimates.

As seen in the graphs DVHop is not affected by ranging errors but all others are affected being DVDistance and Euclidean more susceptible to ranging errors than n-Hop. Euclidean wins in accuracy, followed by DVDistance when there are no ranging errors but for large ranging errors they behave worst than DVHop and n-Hop with location estimation errors of $40 \%$ the radio range. DVHop and $n$-Hop multilateration are equal in acurracy but we must take into account that DVHop is restricted to discrete step values of $h_{s} i z e$ so it's acurracy may be affected by the hop count, that is the sensors density and conectivity. We do not show studies on this factor for space constraints.

The $\mathrm{n}$-Hop multilateration method even more complex and slower than the DVHop does not give any performance increase compared to DVHop. We acknowledge though that we did not implemented the collaborative subtree in the first stage of the iterative process and this may increase overall performance by not adding ill formed nodes to the iterative estimation problem. The euclidean method is also complex and in sparse networks may be unable to estimate location of all nodes in the network reducing coverage but for all the scenarios we simulated both n-Hop and Euclidean where able to converge fast even n-Hop was able to locate all nodes without using collaborative multilateration for anchor nodes densities larger than ten. This follows the fact that anchor nodes at the border of the network increases the reliability of the localization methods.

One advantage of Euclidean and n-Hop over DVhop and DVDistance is that they use direct distances to anchor nodes to estimate new positions. This has the effect that even when the network is not isotropic and the hop count between anchors and sensor nodes do not approximates the true distance the Euclidean and n-Hop methods manage to get good location estimates.

\section{DISCUSSION}

We see from the previous results that all localization methods are affected by different factors. For example DVDistance is affected greatly by ranging errors while DVHop is not
TABLE II

SIMULATION RESULTS SUMMARY

\begin{tabular}{|l|c|c|c|}
\hline Perfotmance & Range Enor & Accuracy & Complexity \\
\hline worst & DVDistance & DVHop & Euclidean \\
\hline bad & Euclidean & n-Hop & n-Hop \\
\hline good & n-Hop & DVDistance & DVHop \\
\hline best & DVHop & Euclidean & DVDistance \\
\hline
\end{tabular}

affected at all. On the other hand DVHop is less accurate than DVDistance and Euclidean for low ranging errors. $n$ Hop multilateration performes as the DVHop method but still DVHop is far more simple to implement and deploy. In table II we summarize this results.

The family of methods proposed by Niculescu seem to be fit for deployment under our requirements since each method compensates the other. DVHop can be used when no ranging information is available, if ranging information is available we could use DVDistance to get better accurate estimates and if the network is not isotropic we may use Euclidean.

The combination of this three methods outperforms the n-Hop Multilateration method but still we could use n-Hop instead of Euclidean when the network is not isotropic and ranging errors are too high. We also noted that Euclidean was the only method that had nodes with no location estimates at the end of the simulation, specially for low anchor node densities causing the overall error estimate to increase. In Euclidean the conditions to be able to infer oistances anchor nodes are more strict than other approaches, this resulted in several nodes without enough distance estimates to anchor nodes to perform the min max localization. This makes Euclidean inadequate for networks with low number of neighbors per node (low connectivity) and low anchor nodes densities.

One last point we must mention is that of communication overhead. It is always mention that sensor networks have lim. ited processing and energy capacity and that communication is the operation that consumes most of the energy. DVHop is has large communication requirements because of the average hop size correction it uses to estimate distances. We argue that this communication costs can be greatly reduced using more efficient broadcasting mechanisms. Until now al! localization methods use controlled broadcasting (Simple Flooding) to propagate distance and location information through the network. We ran the same simulation runs for the DVHop localization method with 60 sensor node, 70 anchor nodes and $0 \%$ ranging error using both Controlled Flooding and Counter Based Flooding. The localization estimation results where equal for both cases and the coverage was not affected, but with the Counter Based Broadcasting we were able to reduce the communication overhead over $75 \%$ as shown in figure 8.

Is important to note that Counter Based Broadcasting is better than Controlled Flooding but it is far from being the best broadcasting algorithm. More advanced broadcasting mechanisms based on topology may further reduce the communication costs of the localization algorithms without 
affecting the location estimation or coverage of the localization methods.

\section{CONCLUSIONS AND FUTURE RESEARCH}

The use of wireless sensor networks in emergency and disaster relief operations promise to provide real time information of the disaster area to rescue teams making coordination and planning more effective. Location information of victims, rescuers and objects in the disaster is vital for the rescue operations. We evaluated several localization methods for wireless sensor networks proposed in the literature and found the family of protocols by Niculescu to fit well the requirements we outlined for deployment in a disaster area Simulation results show that this localization methods do not compete between them, they complement each other; where one method fails the other can take care.

The results of this work are no different from what others have reported. Our contribution is the evaluation of localization methods under certain conditions and for a specific application. We found that under this conditions the expected behavior of the localization methods vary, for example increasing the anchor node density bas little, if not at all, effect on the localization accuracy, contrary to what others have reported. Also the use of anchor nodes at the edges of the perimeter allowed use to make some assumptions that greatly simplify the algorithmic complexity and implementation of the localization methods

The DVHop family of localization methods can be used in a combined manner in all situations and network configurations and conditions. $\mathbf{n}$-Hop multilateration keeps itself in between all three DV methods in accuracy, complexity and ranging robustness. Centralized methods and Centroid based methods where found inappropriate for our requirements and were not considered for simulation. The use of adaptive algorithms that deploy different localization methods depending on the conditions of the network is a topic of research in our agenda.

We also stress the need for more studies of localization and other wireless sensor networks protocols in specific application domains and environments. This may lead to optimizations of the algorithms to specific environments that may improve the overall performance of the localization methods in that specific areas.

\section{REFERENCES}

[1] L. Doherty, K. Pister, and L. El Ghaoui, Comex position estimation in wireless sensor networks, In Proceedings of the 20st Annual Joint Conference of the IEEE Computer and Communications Societies (INFOCOM20), pp-1655-1663, Anchorage, AK, April 2001.

[2] D. Niculescu and B. Nath, Ad Hoc Positioning System (APS), IEEE GLOBECOM 200], San Antonio, Nov 2001

[3] N. Bulusu, J. Heidemann, and D. Estrin, GPS-less towcost outdoo lacalization for very small devices, IEEE Personal Communications $7(5): 28-34$, Oct 2000 .

[4] C. Savarese, J, Rabacy, and J. Bentel, Locationing in Distributed Ad-Hoc Wireless Sensor Networks, In IEEE lnternation Conference on Acoustics, Speech, and Signal Processing (ICASSP), pages 20037-2040, Salt Lake City, UT, May 2001

[5] A. Savvides, C.C. Han, and M. Srivastava, Dymanic fine-grained localization in ad-hoc networks of sensors, In 7th ACM Intemational Conference on Mobile Computing and Networking (MOBICOM), pages 166-179, Rome. Italy, July 200
[6] B. Williams and T. Camp, Comparison of Broadcasting Techniques for Mobile Ad Hoc Networks, Proceedings of the ACM International Symposium on Mobile Ad Hoc Networking and Computing (MOB[HOC '02), pp.194-205, 2002.

[7] A. Savvides, H. Park, and M. Srivastava, The bits and flops of the $N$ hop multilateration primitive for node localization problems, First ACM International Workshop on Wireless Sensor Networks and Applications (WSNA), Allanta, GA, 2002, pp. 112-121

[8] R. Nagpal, H. Shrobe, and J. Bachrach, Organizing a Global Coordinate System from Local Information on an Ad Hoc Sensor Nenwork. In the 2nd International Workshop on Information Processing in Sensor Networks (IPSN '03), Palo Alto, April, 2003

[9] L. Hu and D. Evans. Localization for Mobile Sensor Nenworks, Tenth Annual International Conference on Mobile Computing and Networking (ACM MobiCom 2004). 26 September - 1 October 2004.

[10] $\mathrm{X} . \mathrm{Ji}$ and $\mathrm{H}$. Zha, Mwidimensional scaling based sensor positioning algorithms in wireless sensor networks, In Proceedings of the 1th Annual ACM Conference on Embedded Networked Sensor Systems, pp.328-329, November, 2003.

[11] A. Vargas, The OMNet++ Discrete Event Sirrulator, http://waw.onnetpp.org

[12] WERT, Wineless Emergency Response Team: Final Report, for the September 11,2001 New York City World Trade Center Terrorist Attack, October 2001

[13] T. He, C. Huang, B.M. Blum,3. A. Stankovic,and T, F. Abdelzaher. Range-Free Localization Schemes in Large Scale Sensor Networks, CSTR2003 -06. Submit to MobiCom 2003.

[14] L. Kleinrock and J. Silvester. Optimum transmission radii for packet radio networks ar why six is a magic number. Proc. Natnl. Telecornm. Conf, pages 4.3.I-4.3.5, 1978

[15] K . Langendoen and N. Reijers, Distributed localization in wireless sensor networks: a quantitative comparison., Computer Networks: The International Journal of Computer and Telecommunications Networking, pages 499-518, November 2003.

[16] B. Archana and P. Vijay.Sensor Networks: An Overview,Department of Computer Science, University of Califorria, Davis

[17] $M$. Andreas and $L$ Thomas and $R$. Thomas and $K$. Thomas and K. Holger. Design Challenges for an Integrated Disaster Management Communication and Informasion System, The First IEEE Workshop on Disaster Recovery Networks (DIREN 2002), IEEE INFOCOM 2002, New York City, June 24, 2002. 